\section{Special Issue on Differential Geometry \\ - ARTICLES •}

July 2021 Vol. 64 No. 7: 1373-1390

https://doi.org/10.1007/s11425-020-1744-9

\title{
Harmonic maps with torsion
}

\author{
In Memory of Professor Zhengguo Bai (1916-2015) \\ Volker Branding \\ Faculty of Mathematics, University of Vienna, Vienna 1090, Austria \\ Email:volker.branding@univie.ac.at
}

Received February 25, 2020; accepted July 16, 2020; published online December 3, 2020

\begin{abstract}
In this article we introduce a natural extension of the well-studied equation for harmonic maps between Riemannian manifolds by assuming that the target manifold is equipped with a connection that is metric but has non-vanishing torsion. Such connections have already been classified in the work of Cartan (1924). The maps under consideration do not arise as critical points of an energy functional leading to interesting mathematical challenges. We will perform a first mathematical analysis of these maps which we will call harmonic maps with torsion.
\end{abstract}

Keywords harmonic maps with torsion, metric torsion, regularity of weak solutions

MSC(2020) 58E20, 53C43

Citation: Branding V. Harmonic maps with torsion. Sci China Math, 2021, 64: 1373-1390, https://doi.org/ 10.1007/s11425-020-1744-9

\section{Introduction and results}

The harmonic map equation is one of the most studied partial differential equations for maps between Riemannian manifolds. Given a smooth map $\phi$ between two Riemannian manifolds $(M, g)$ and $(N, h)$ the harmonic map equation can be obtained by computing the first variation of the energy of a map which is given by

$$
E(\phi)=\int_{M}|d \phi|^{2} \mathrm{dvol}_{g}
$$

The critical points of (1.1) are characterized by the vanishing of the so-called tension field

$$
0=\tau(\phi):=\operatorname{Tr}_{g} \nabla^{\phi^{*} T N} d \phi
$$

The (standard) harmonic map equation (1.2) is a semilinear second order elliptic partial differential equation for which many results on existence and qualitative behavior of its solutions could be achieved over the years. For an overview on the current status of research we refer to the survey article [20].

In the literature on harmonic maps one usually chooses to utilize the Levi-Civita connection on the target manifold $N$. If one defines harmonic maps via a variational principle, as we have done above, then, 
as we will see later, the variational approach chooses to employ the Levi-Civita connection on the target manifold.

This article is devoted to a first study of harmonic maps that are coupled to a torsion endomorphism on the target manifold. Harmonic maps with a connection different from the Levi-Civita connection on the domain manifold have already been investigated in great generality. Such kind of maps became known as $V$-harmonic maps and includes the classes of Hermitian, affine and Weyl harmonic maps into Riemannian manifolds; see the introduction of [15] for more details.

In order to obtain a generalization of the harmonic map equation that takes into account a connection with metric torsion on the target manifold we have to abandon the variational point of view. Although this leads to a straightforward generalization of the standard harmonic map equation (1.2) the non-variational nature of harmonic maps with torsion leads to severe technical difficulties.

The central equation studied in this article is

$$
\tau^{\text {Tor }}(\phi):=\tau(\phi)+A(d \phi, d \phi)=0,
$$

where the superscript "Tor" represents that we are considering a connection with torsion. The nonvanishing torsion is given by the torsion endomorphism $A(d \phi, d \phi)$ on which we will give more details later.

Solutions of (1.3) will be called harmonic maps with torsion. Equation (1.3) can be obtained by taking the standard harmonic map equation and then changing to a connection with metric torsion.

The energy of a map (1.1) is invariant under various symmetry operations as for example the invariance under diffeomorphisms on the domain. By Noether's theorem these invariances all lead to a conserved quantity which can then be successfully applied in the analysis of the harmonic map equation.

The heat flow method is a strong tool in the analysis of the standard harmonic map equation that allows one to derive various existence results (see [23] for an overview). Unfortunately, it seems that this method is no longer applicable (at least without making many additional assumptions) in the presence of torsion. One should expect such a phenomenon: The heat flow for standard harmonic maps decreases the energy of a map (1.1) and tries to flow to a critical point. As there does not exist an energy functional for harmonic maps with torsion the heat flow cannot find a direction in which it decreases the energy and we cannot expect the gradient flow to converge.

While connections with metric torsion have been intensively studied in the physics literature, so far, not many mathematicians have taken up this direction of research. Let us mention several mathematical results that are connected to the equation studied in this article.

(1) Geodesics with vectorial torsion were studied in [4]. Vectorial torsion comprises a particular kind of torsion endomorphism in (1.3).

(2) Dirac-harmonic maps with torsion, which are a mathematical version of a model from supersymmetric quantum field theory, have been investigated in [6].

(3) VT-harmonic maps [15] are solutions of the following equation:

$$
\tau(\phi)+d \phi(V)+\operatorname{Tr}_{g} T(d \phi, d \phi)=0 .
$$

Here, $V \in \Gamma(T M)$ and $T$ is a $(2,1)$-tensor on $T N$. Although the form of $V T$-harmonic maps is very similar to harmonic maps with torsion it seems that the additional structure in (1.3) arising due to the torsion on $N$ leads to a rich mathematical framework.

(4) Magnetic harmonic maps $[8,9]$ from a two-dimensional domain share some similarities with (1.3). They arise as critical points of

$$
E_{B}(\phi)=\int_{M}\left(|d \phi|^{2}+\frac{1}{2} \phi^{*} B\right) \mathrm{dvol}_{g}
$$

where $B$ is a two-form on $N$ and $\operatorname{dim} N \geqslant 3$. The critical points of (1.5) are given by

$$
\tau(\phi)=Z\left(d \phi\left(e_{1}\right) \wedge Z d \phi\left(e_{2}\right)\right)
$$


and it is obvious that they have a structure analogous to harmonic maps with torsion (1.3). Here, $\left\{e_{1}, e_{2}\right\}$ is an orthonormal basis of $T M$ and the vector-bundle homomorphism $Z \in \Gamma\left(\operatorname{Hom}\left(\Lambda^{2} T^{*} N, T N\right)\right)$ is defined by the equation

$$
\Omega\left(\eta, \xi_{1}, \xi_{2}\right)=\left\langle Z\left(\xi_{1} \wedge \xi_{2}\right), \eta\right\rangle
$$

where $\Omega=d B$ is a three-form on $N$ and $\xi_{1}, \xi_{2}, \eta \in \Gamma(T N)$.

(5) In the study of pseudoharmonic maps one is also naturally led to consider the case of connections having torsion $[16,27]$. However, these maps still arise from a variational principle and the non-vanishing torsion is given on the domain manifold.

(6) The results of this article may also be interesting in the study of harmonic maps to Lie groups as one can have a non-symmetric connection in the case of a non-abelian Lie group.

In the case of $N=\mathbb{R}^{q}$ with the flat metric the standard harmonic map equation reduces to the linear Laplace equation, whereas the equation for harmonic maps with torsion (1.3) would still be nonlinear due to the non-vanishing torsion.

Throughout this article the notation will be as follows: Local coordinates on $M$ will be denoted by $x^{i}$ whereas for local coordinates on $N$ we will use $y^{\alpha}$. We will use Latin indices $i, j, k=1, \ldots, m:=\operatorname{dim} M$ on the domain manifold $M$ while we will employ Greek letters $\alpha, \beta, \gamma, \ldots, n:=\operatorname{dim} N$ to represent indices on the target manifold $N$. Moreover, we will make use of the Einstein summation convention and tacitly sum over repeated indices.

The rest of this article is organized as follows. In Section 2, we provide the necessary background on metric connections with torsion and study the equation for harmonic maps with torsion in more detail. Section 3 is devoted to geometric aspects of harmonic maps with torsion. We derive various Bochner formulas, study the effects of conformal transformations on harmonic maps with torsion and also discuss their stability. In Section 4, we study several analytic aspects of harmonic maps with torsion. We show that they satisfy the unique continuation property, study the regularity of weak solutions, prove a removable singularity theorem and finally provide a Liouville type result under a small energy assumption.

We will mostly apply techniques from standard harmonic maps in our analysis as far as they are still applicable.

Throughout this article we will assume that the manifold $M$ is closed, i.e., compact and without boundary, unless explicitly stated otherwise.

\section{Harmonic maps with torsion}

Before we turn our attention to harmonic maps coupled to torsion, let us give a short introduction to connections with metric torsion, where we follow the introduction of [4].

\subsection{A shortcut to connections with metric torsion}

We consider a Riemannian manifold $(N, h)$ and by $\nabla^{\mathrm{LC}}$ we denote its Levi-Civita connection. For any affine connection there exists a $(2,1)$-tensor field $A$ such that

$$
\nabla_{X} Y=\nabla_{X}^{\mathrm{LC}} Y+A(X, Y)
$$

for all vector fields $X, Y \in \Gamma(T N)$.

We require that the connection $\nabla$ is orthogonal, i.e., for all the vector fields $X, Y, Z$ we have

$$
\partial_{X}\langle Y, Z\rangle=\left\langle\nabla_{X} Y, Z\right\rangle+\left\langle Y, \nabla_{X} Z\right\rangle
$$

where $\langle\cdot, \cdot\rangle$ denotes the scalar product of the metric $h$. By combining (2.1) and (2.2) it follows that the endomorphism $A(X, \cdot)$ has to be skew-adjoint

$$
\langle A(X, Y), Z\rangle=-\langle Y, A(X, Z)\rangle .
$$


Any torsion tensor $A$ induces a $(3,0)$-tensor via the assignment

$$
A_{X Y Z}=\langle A(X, Y), Z\rangle .
$$

We define the space of all the admissible torsion tensors on $T_{p} N$ by

$$
\mathcal{T}\left(T_{p} N\right):=\left\{A \in \otimes^{3} T_{p}^{*} N \mid A_{X Y Z}=-A_{X Z Y}, X, Y, Z \in T_{p} N\right\} .
$$

For $A \in \mathcal{T}\left(T_{p} M\right)$ and $Z \in T_{p} M$ we set

$$
c_{12}(A)(Z)=A_{\partial_{y^{i}} \partial_{y^{i}} Z},
$$

where $\partial_{y^{i}}$ is an orthonormal basis of $T N$. Metric connections with torsion have been classified by Cartan [14] who proved the following theorem.

Theorem 2.1 (See [14]). Assume that $\operatorname{dim} N \geqslant 3$. Then the space $\mathcal{T}\left(T_{p} N\right)$ has the following irreducible decomposition:

$$
\mathcal{T}\left(T_{p} N\right)=\mathcal{T}_{1}\left(T_{p} N\right) \oplus \mathcal{T}_{2}\left(T_{p} N\right) \oplus \mathcal{T}_{3}\left(T_{p} N\right),
$$

which is orthogonal with respect to $\langle\cdot, \cdot\rangle$ and is explicitly given by

$$
\begin{aligned}
& \mathcal{T}_{1}\left(T_{p} N\right)=\left\{A \in \mathcal{T}\left(T_{p} N\right) \mid \exists V \text { such that } A_{X Y Z}=\langle X, Y\rangle\langle V, Z\rangle-\langle X, Z\rangle\langle V, Y\rangle\right\}, \\
& \mathcal{T}_{2}\left(T_{p} N\right)=\left\{A \in \mathcal{T}\left(T_{p} N\right) \mid A_{X Y Z}=-A_{Y X Z}, \forall X, Y, Z\right\} \\
& \mathcal{T}_{3}\left(T_{p} N\right)=\left\{A \in \mathcal{T}\left(T_{p} N\right) \mid A_{X Y Z}+A_{Y Z X}+A_{Z X Y}=0 \text { and } c_{12}(A)(Z)=0\right\}
\end{aligned}
$$

Moreover, for $\operatorname{dim} N=2$ we have

$$
\mathcal{T}\left(T_{p} N\right)=\mathcal{T}_{1}\left(T_{p} N\right)
$$

For a proof of the above theorem we refer to [34, Theorem 3.1].

Corollary 2.2. For any orthogonal connection on a Riemannian manifold $N$ and $\operatorname{dim} N \geqslant 3$ the torsion tensor can be written as

$$
A(X, Y)=\langle X, Y\rangle V-\langle V, Y\rangle X+T(X, Y, \cdot)^{\sharp}+S(X, Y, \cdot)^{\sharp},
$$

where $T(X, Y, \cdot)^{\sharp}$ and $S(X, Y, \cdot)^{\sharp}$ are uniquely defined via

$$
T(X, Y, Z)=\left\langle T(X, Y, \cdot)^{\sharp}, Z\right\rangle, \quad S(X, Y, Z)=\left\langle S(X, Y, \cdot)^{\sharp}, Z\right\rangle .
$$

Here, we have $T(X, Y, Z) \in \tau_{2}\left(T_{p} N\right)$ and $S(X, Y, Z) \in \tau_{3}\left(T_{p} N\right)$.

We will use the following terminology: A torsion endomorphism is called

(1) vectorial if it is contained in $\mathcal{T}_{1}\left(T_{p} N\right)$,

(2) totally antisymmetric if it lies in $\mathcal{T}_{2}\left(T_{p} N\right)=\Lambda^{3} T_{p}^{*} N$,

(3) of Cartan type if it is an element of $\mathcal{T}_{3}\left(T_{p} N\right)$.

Throughout this article we make use of the following sign convention for the curvature of a connection:

$$
R(X, Y) Z=\nabla_{X} \nabla_{Y} Z-\nabla_{Y} \nabla_{X} Z-\nabla_{[X, Y]} Z
$$

for given vector fields $X, Y, Z$.

We have the following relation between the curvature tensors of $\nabla^{\text {Tor }}$ and $\nabla^{\mathrm{LC}}$ :

$$
\begin{aligned}
R^{\mathrm{Tor}}(X, Y) Z= & R^{\mathrm{LC}}(X, Y) Z+\left(\nabla_{X}^{\mathrm{LC}} A\right)(Y, Z)-\left(\nabla_{Y}^{\mathrm{LC}} A\right)(X, Z) \\
& +A(X, A(Y, Z))-A(Y, A(X, Z)) .
\end{aligned}
$$

It is obvious that the curvature tensor of a connection with metric torsion is antisymmetric in $X, Y$ as is the standard Riemann curvature tensor. However, not all of the symmetries of the Riemann curvature tensor still hold true in the presence of non-vanishing torsion. 
The torsion tensor $T(X, Y)$ is related to the torsion endomorphism $A(X, Y)$ via

$$
\nabla_{X} Y-\nabla_{Y} X-[X, Y]=T(X, Y)=A(X, Y)-A(Y, X)
$$

for given vector fields $X, Y$.

The particular structure of the torsion endomorphism in (2.4) will have a significant influence on the geometry of the manifold $N$.

There exist several geometric settings in which connections with torsion naturally appear. Consider a compact Hermitian manifold $M$ with metric $g$, complex structure $J$ and Kähler form $\omega$. It has a canonical connection, the so-called Chern connection, defined by $\nabla g=0$ and $\nabla J=0$, which has non-vanishing torsion (see, for example, [24]).

Another famous connection in Hermitian geometry that carries non-vanishing torsion is the Gauduchon connection (see, for example, $[18,19]$ ).

In the case of a pseudo-Hermitian manifold, there exists a canonical linear connection which preserves both the CR structure and the Webster metric. This particular connection is called the Tanaka-Webster connection (see [33,35] and also [17, Subsection 1.2] for more details), and it also has non-vanishing torsion.

Another example where a connection with torsion naturally appears in differential geometry is the following: The reduction of the structure group of a Riemannian manifold leads to a connection with torsion adapted to the associated geometric structure (see, for example, [2, Subsection 4.2] for more details).

In physics, one often chooses to consider totally antisymmetric torsion as in this case the geodesics are the same as for the Levi-Civita connection. For a summary on metric connections with torsion applied in physics we refer to [32]. A mathematical analysis of connections with totally antisymmetric torsion as they are applied in string theory was carried out in [1]. A general discussion on spin geometry and the Dirac operator associated with a metric connection with torsion can be found in [26].

The geodesics of connections with vectorial torsion were investigated in [4], and various geometric aspects of manifolds having a connection with vectorial torsion were studied in [3]. The uniformization theorem on closed surfaces for a metric connection with vectorial torsion was proved via the Ricci flow in [13].

Up to the best knowledge of the author a systematic study of Riemannian manifolds equipped with a metric connection with Cartan type torsion has not been performed.

For more details on the geometry of Riemannian manifolds having a connection with metric torsion we refer to the lecture notes [2].

\subsection{Harmonic maps with torsion}

The way we have obtained the equation for harmonic maps with torsion (1.3) was considering the standard harmonic map equation and passing over to a connection with metric torsion on the target manifold $N$.

We would like to emphasize once more that harmonic maps with torsion are non-variational and cannot be obtained as a critical point of an energy functional in general.

More precisely, if we consider a variation of the map $\phi$ given by $\phi_{t}: M \times(-\epsilon, \epsilon) \rightarrow N$ satisfying $\left.\frac{\partial \phi_{t}}{\partial t}\right|_{t=0}=\eta$, where $\epsilon>0$ is a small number, a direct calculation shows

$$
\begin{aligned}
\left.\frac{d}{d t}\right|_{t=0} \frac{1}{2} \int_{M}\left|d \phi_{t}\right|^{2} \mathrm{dvol}_{g} & =\left.\int_{M}\left\langle\frac{\nabla^{\mathrm{Tor}}}{d t} d \phi_{t}\left(e_{i}\right), d \phi_{t}\left(e_{i}\right)\right\rangle \mathrm{dvol}_{g}\right|_{t=0} \\
& =\left.\int_{M}(\left\langle\frac{\nabla^{\mathrm{LC}}}{d t} d \phi_{t}\left(e_{i}\right), d \phi_{t}\left(e_{i}\right)\right\rangle+\underbrace{\left\langle A\left(d \phi_{t}\left(\partial_{t}\right), d \phi_{t}\right), d \phi_{t}\right\rangle}_{=-\left\langle A\left(d \phi_{t}\left(\partial_{t}\right), d \phi_{t}\right), d \phi_{t}\right\rangle=0}) \mathrm{d} \operatorname{vol}_{g}\right|_{t=0} \\
& =\left.\int_{M}\left\langle\nabla_{e_{i}}^{\mathrm{LC}} d \phi_{t}\left(\partial_{t}\right), d \phi_{t}\left(e_{i}\right)\right\rangle \mathrm{dvol}_{g}\right|_{t=0} \\
& =-\int_{M}\langle\eta, \tau(\phi)\rangle \operatorname{dvol}_{g}
\end{aligned}
$$


confirming the fact that the critical points of the Dirichlet energy are standard harmonic maps even if we consider a connection with torsion on the target.

Let us now analyze the structure of the torsion endomorphism in (1.3) in more detail. Using the decomposition of the torsion endomorphism given in (2.4) we find

$$
A(d \phi, d \phi)=|d \phi|^{2} V-\langle V, d \phi\rangle d \phi+T(d \phi, d \phi, \cdot)^{\sharp}+S(d \phi, d \phi, \cdot)^{\sharp} .
$$

Note that due to symmetry reasons the totally antisymmetric part of the torsion does not contribute to $(1.3)$ as we have

$$
\begin{aligned}
T(d \phi, d \phi, \cdot)^{\sharp} & =T\left(d \phi\left(e_{i}\right), d \phi\left(e_{j}\right), \cdot\right)^{\sharp} g^{i j} \\
& =-T\left(d \phi\left(e_{j}\right), d \phi\left(e_{i}\right), \cdot\right)^{\sharp} g^{j i} \\
& =-T(d \phi, d \phi, \cdot)^{\sharp} \\
& =0 .
\end{aligned}
$$

Hence, in general, the equation for a harmonic map with torsion acquires the form

$$
\tau(\phi)+|d \phi|^{2} V-\langle V, d \phi\rangle d \phi+S(d \phi, d \phi, \cdot)^{\sharp}=0 .
$$

In the case of a two-dimensional target only the vectorial torsion contributes in (1.3) and the equation for harmonic maps with torsion is further simplified to

$$
\tau(\phi)+|d \phi|^{2} V-\langle V, d \phi\rangle d \phi=0 .
$$

By choosing local coordinates $\left(U, x^{i}\right)$ on $M$ and local coordinates $\left(V, y^{\alpha}\right)$ on $N$ such that $\phi(U) \subset V$, the equation for harmonic maps with torsion acquires the form

$$
\Delta \phi^{\alpha}+\Gamma_{\beta \gamma}^{\alpha}(\phi) \frac{\partial \phi^{\beta}}{\partial x^{i}} \frac{\partial \phi^{\gamma}}{\partial x^{j}} g^{i j}+A_{\beta \gamma}^{\alpha}(\phi) \frac{\partial \phi^{\beta}}{\partial x^{i}} \frac{\partial \phi^{\gamma}}{\partial x^{j}} g^{i j}=0,
$$

where $\alpha=1, \ldots, \operatorname{dim} N$.

For most of the computations carried out in this article the precise structure of the torsion endomorphism $A(X, Y)$ will not be important and we will mainly work with (1.3).

\subsection{Geodesics with torsion}

As in the case of standard geodesics it is straightforward to see that geodesics with torsion have constant speed. Let $\gamma: I \subset \mathbb{R} \rightarrow N$ be a curve and denote the derivative with respect to the curve parameter by '.

Lemma 2.3. Let $\gamma: I \rightarrow N$ be a solution of (1.3). Then $\left|\gamma^{\prime}\right|^{2}$ is constant.

Proof. We compute

$$
\frac{d}{d s}\left|\gamma^{\prime}\right|^{2}=2\left\langle\frac{\nabla^{\mathrm{LC}}}{d s} \gamma^{\prime}, \gamma^{\prime}\right\rangle=2\left\langle\tau(\gamma), \gamma^{\prime}\right\rangle=-2\left\langle A\left(\gamma^{\prime}, \gamma^{\prime}\right), \gamma^{\prime}\right\rangle=0,
$$

where we first used (1.3) and afterwards the skew-adjointness of the torsion endomorphism completing the proof.

This statement also holds true for magnetic geodesics [12] which have some similarity with geodesics with torsion. In particular, magnetic geodesics also do not always arise from a variational principle leading to the same technical difficulties as outlined in this article.

In terms of coordinates the equation for geodesics with torsion is given by

$$
\gamma^{\prime \prime \alpha}=-\left(\Gamma_{\beta \delta}^{\alpha}(\gamma)+A_{\beta \delta}^{\alpha}(\gamma)\right) \gamma^{\prime \beta} \gamma^{\prime \delta} .
$$

If we interpret this equation as an ordinary differential equation then we know that we can always extend a solution beyond a given interval of existence as the right-hand side is bounded. However, we cannot expect to find a generalization of the Hopf-Rinow theorem from Riemannian geometry as was demonstrated in [4, Section 4]. 


\section{Geometric aspects of harmonic maps with torsion}

In this section we study various geometric aspects related to harmonic maps with torsion.

\subsection{Bochner formulas}

The Bochner technique is a fundamental tool in the analysis of geometric partial differential equations. It is based on interchanging covariant derivatives and making use of the resulting curvature terms in order to get a deeper understanding of the solution space of the corresponding geometric partial differential equation.

In the case of harmonic maps with torsion we can make use of the Bochner technique based on either the Levi-Civita connection or a metric connection with torsion.

Throughout this section we choose a local orthonormal basis around a point $p \in M$ such that $\nabla_{e_{i}} e_{j}=0$ at $p$ for $i, j=1, \ldots, \operatorname{dim} M$.

Let us first recall the Bochner formula for standard harmonic maps obtained by using the Levi-Civita connection, i.e.,

$$
\Delta^{\mathrm{LC}} d \phi\left(e_{j}\right)=d \phi\left(\operatorname{Ric}^{M}\left(e_{j}\right)\right)+R^{N}\left(d \phi\left(e_{i}\right), d \phi\left(e_{j}\right)\right) d \phi\left(e_{i}\right)+\nabla_{e_{j}}^{\mathrm{LC}} \tau(\phi) .
$$

For a proof of (3.1) we refer to [36, Proposition 1.34].

Using the Bochner formula for the Levi-Civita connection (3.1) we obtain

$$
\begin{aligned}
\Delta \frac{1}{2}|d \phi|^{2}= & \left|\nabla^{\mathrm{LC}} d \phi\right|^{2}+\left\langle d \phi\left(\operatorname{Ric}^{M}\right), d \phi\right\rangle-\left\langle R^{N}\left(d \phi\left(e_{i}\right), d \phi\left(e_{j}\right)\right) d \phi\left(e_{j}\right), d \phi\left(e_{i}\right)\right\rangle \\
& +\left\langle\nabla_{e_{j}}^{\mathrm{LC}} \tau(\phi), d \phi\left(e_{j}\right)\right\rangle .
\end{aligned}
$$

One can deduce with the help of the maximum principle that if $M$ has positive Ricci curvature and $N$ non-positive sectional curvature then every harmonic map must be constant. In the following we want to analyze if this statement remains true in the presence of torsion on $N$.

To this end, we derive a generalization of the Bochner formula (3.1) taking into account the nonvanishing torsion.

Proposition 3.1 (Bochner formula with torsion). Let $\phi: M \rightarrow N$ be a smooth map and suppose that $N$ is equipped with a connection with non-vanishing torsion. Then the following Bochner formula holds:

$$
\begin{aligned}
\Delta^{\mathrm{Tor}} d \phi\left(e_{j}\right)= & d \phi\left(\operatorname{Ric}^{M}\left(e_{j}\right)\right)+R_{\text {Tor }}^{N}\left(d \phi\left(e_{i}\right), d \phi\left(e_{j}\right)\right) d \phi\left(e_{i}\right)+\nabla_{e_{j}}^{\mathrm{Tor}} \tau^{\text {Tor }}(\phi) \\
& +\left(\nabla_{d \phi\left(e_{i}\right)}^{\mathrm{LC}} A\right)\left(d \phi\left(e_{i}\right), d \phi\left(e_{j}\right)\right)+A\left(\tau(\phi), d \phi\left(e_{j}\right)\right) \\
& +A\left(d \phi\left(e_{i}\right), \nabla_{e_{i}}^{\mathrm{LC}} d \phi\left(e_{j}\right)\right)+A\left(d \phi\left(e_{i}\right), A\left(d \phi\left(e_{i}\right), d \phi\left(e_{j}\right)\right)\right) \\
& -\left(\nabla_{d \phi\left(e_{i}\right)}^{\mathrm{LC}} A\right)\left(d \phi\left(e_{j}\right), d \phi\left(e_{i}\right)\right)-A\left(\nabla_{e_{i}}^{\mathrm{LC}} d \phi\left(e_{j}\right), d \phi\left(e_{i}\right)\right) \\
& -A\left(d \phi\left(e_{j}\right), \tau(\phi)\right)-A\left(d \phi\left(e_{i}\right), A\left(d \phi\left(e_{j}\right), d \phi\left(e_{i}\right)\right)\right) .
\end{aligned}
$$

Proof. By a direct calculation we find the following relation between the connection Laplacians for the Levi-Civita connection $\Delta^{\mathrm{LC}}$ and for a connection with torsion $\Delta^{\text {Tor }}$ on $\phi^{*} T N$ :

$$
\begin{aligned}
\Delta^{\mathrm{Tor}} d \phi\left(e_{j}\right)= & \Delta^{\mathrm{LC}} d \phi\left(e_{j}\right)+2 A\left(d \phi, \nabla^{\mathrm{LC}} d \phi\left(e_{j}\right)\right)+\left(\nabla_{d \phi\left(e_{i}\right)}^{\mathrm{LC}} A\right)\left(d \phi\left(e_{i}\right), d \phi\left(e_{j}\right)\right) \\
& +A\left(\tau(\phi), d \phi\left(e_{j}\right)\right)+A\left(d \phi, A\left(d \phi, d \phi\left(e_{j}\right)\right)\right) .
\end{aligned}
$$

Moreover, we have

$$
\begin{aligned}
\nabla_{e_{j}}^{\mathrm{Tor}} \tau^{\mathrm{Tor}}(\phi)= & \nabla_{e_{j}}^{\mathrm{LC}} \tau(\phi)+\left(\nabla_{d \phi\left(e_{j}\right)}^{\mathrm{LC}} A\right)(d \phi, d \phi)+A\left(\nabla_{e_{j}}^{\mathrm{LC}} d \phi, d \phi\right)+A\left(d \phi, \nabla_{e_{j}}^{\mathrm{LC}} d \phi\right) \\
& +A\left(d \phi\left(e_{j}\right), \tau(\phi)\right)+A\left(d \phi\left(e_{j}\right), A(d \phi, d \phi)\right) .
\end{aligned}
$$

The claim now follows from inserting (3.4), (3.5) and (2.5) into the Bochner formula for the Levi-Civita connection (3.1). 
Lemma 3.2. Let $\phi: M \rightarrow N$ be a smooth harmonic map with torsion. Then the following formula holds:

$$
\begin{aligned}
\Delta \frac{1}{2}|d \phi|^{2}= & \left|\nabla^{\mathrm{LC}} d \phi\right|^{2}+\left\langle d \phi\left(\operatorname{Ric}^{M}\right), d \phi\right\rangle-\left\langle R^{N}\left(d \phi\left(e_{i}\right), d \phi\left(e_{j}\right)\right) d \phi\left(e_{j}\right), d \phi\left(e_{i}\right)\right\rangle \\
& -\left\langle\left(\nabla_{d \phi\left(e_{j}\right)}^{\mathrm{LC}} A\right)(d \phi, d \phi), d \phi\left(e_{j}\right)\right\rangle-\left\langle A\left(d \phi\left(e_{i}\right), \nabla_{e_{j}}^{\mathrm{LC}} d \phi\left(e_{i}\right)\right), d \phi\left(e_{j}\right)\right\rangle .
\end{aligned}
$$

Proof. Using that $\phi$ is a smooth solution of (1.3) we calculate

$$
\begin{aligned}
\nabla_{e_{j}}^{\mathrm{LC}} \tau(\phi) & =-\nabla_{e_{j}}^{\mathrm{LC}}(A(d \phi, d \phi)) \\
& =-\left(\nabla_{d \phi\left(e_{j}\right)}^{\mathrm{LC}} A\right)(d \phi, d \phi)-A\left(\nabla_{e_{j}}^{\mathrm{LC}} d \phi\left(e_{i}\right), d \phi\left(e_{i}\right)\right)-A\left(d \phi\left(e_{i}\right), \nabla_{e_{j}}^{\mathrm{LC}} d \phi\left(e_{i}\right)\right) .
\end{aligned}
$$

Note that

$$
\begin{aligned}
\left\langle A\left(\nabla_{e_{j}}^{\mathrm{LC}} d \phi\left(e_{i}\right), d \phi\left(e_{i}\right)\right), d \phi\left(e_{j}\right)\right\rangle & =\left\langle A\left(\nabla_{e_{i}}^{\mathrm{LC}} d \phi\left(e_{j}\right), d \phi\left(e_{i}\right)\right), d \phi\left(e_{j}\right)\right\rangle \\
& =-\left\langle A\left(\nabla_{e_{i}}^{\mathrm{LC}} d \phi\left(e_{j}\right), d \phi\left(e_{j}\right)\right), d \phi\left(e_{i}\right)\right\rangle \\
& =-\left\langle A\left(\nabla_{e_{j}}^{\mathrm{LC}} d \phi\left(e_{i}\right), d \phi\left(e_{i}\right)\right), d \phi\left(e_{j}\right)\right\rangle=0,
\end{aligned}
$$

where we first used the symmetry of the Levi-Civita connection and the skew-symmetry of the torsion endomorphism in the second step. Inserting the above equality into (3.2) completes the proof.

Remark 3.3. $\quad$ It seems rather difficult to extract more information from (3.6) due to the presence of the torsion terms.

\subsection{The effects of conformal transformations}

In this subsection we want to understand the effects of conformal transformations on both the domain and the target manifold in the context of harmonic maps with torsion.

In order to analyze the effect of a conformal transformation on the domain let $\phi:(M, g) \rightarrow(N, h)$ be a smooth map. If we perform a conformal transformation of the metric on the domain, i.e., $\tilde{g}=\mathrm{e}^{2 u} g$ for some smooth function $u$, we have the following formula for the transformation of the tension field:

$$
\tau_{\tilde{g}}(\phi)=\mathrm{e}^{-2 u}\left(\tau_{g}(\phi)+(m-2) d \phi(\nabla u)\right),
$$

where $\tau_{\tilde{g}}(\phi)$ denotes the tension field of the map $\phi$ with respect to the metric $\tilde{g}$ and the Levi-Civita connection on $N$.

Moreover, it is easy to check that the torsion endomorphism satisfies

$$
A_{\tilde{g}}(d \phi, d \phi)=\mathrm{e}^{-2 u} A_{g}(d \phi, d \phi),
$$

where the notation $A_{g}(d \phi, d \phi)$ highlights that we are using the metric $g$. Hence, we obtain

$$
\tau_{\tilde{g}}^{\text {Tor }}(\phi)=\mathrm{e}^{-2 u}\left(\tau_{g}^{\text {Tor }}(\phi)+(m-2) d \phi(\nabla u)\right) .
$$

We may conclude that (as in the case of standard harmonic maps) the equation for harmonic maps with torsion (1.3) is invariant under conformal transformations on the domain in the case $\operatorname{dim} M=2$. The invariance under conformal transformations for standard harmonic maps in $\operatorname{dim} M=2$ gives rise to a conserved quantity, i.e., the associated stress-energy tensor is tracefree. However, in order to obtain the stress-energy tensor one needs to have an energy functional at hand which one can vary with respect to the metric on the domain. As harmonic maps with torsion are non-variational we cannot expect to find a generalization of the stress-energy tensor for them and also do not obtain a conserved quantity.

As a second step, we point out an interesting similarity between harmonic maps with vectorial torsion and conformal transformations on the target manifold.

One could ask the natural question: Suppose that we have given a standard harmonic map. Can we deform it into a harmonic map with torsion by performing a conformal transformation of the metric on the target? 
To approach this question let $\tilde{h}=\mathrm{e}^{2 v} h$ be a conformal transformation of the metric on the target manifold, where $v \in C^{\infty}(N)$. Then, for all $X, Y \in \Gamma(T N)$ the Levi-Civita connections of $h$ and $\tilde{h}$ are related via

$$
\tilde{\nabla}_{X}^{\mathrm{LC}} Y=\nabla_{X}^{\mathrm{LC}} Y+X(v) Y+Y(v) X-h(X, Y) \nabla v .
$$

Hence, the tension fields (computed with respect to the Levi-Civita connection) satisfy

$$
\tilde{\tau}^{\mathrm{LC}}(\phi)=\tau^{\mathrm{LC}}(\phi)+2\langle d \phi, \nabla v\rangle d \phi-|d \phi|^{2} \nabla v .
$$

Note that the torsion endomorphism $A(d \phi, d \phi)$ does not depend on the metric on the target and is not affected by a conformal transformation on the target.

Now, recall that in the case of vectorial torsion the equation for harmonic maps with torsion (1.3) acquires the form

$$
\tau(\phi)+|d \phi|^{2} V-\langle V, d \phi\rangle d \phi=0 .
$$

In the case where $V$ is a gradient vector field, i.e., $V=\nabla v$, there is an interesting similarity (up to a factor of two) between (3.7) and (3.8).

\subsection{The stability of harmonic maps with torsion}

In order to understand the stability of standard harmonic maps one usually calculates the second variation of the Dirichlet energy (1.1) for a map between two Riemannian manifolds and evaluates it at a critical point (see, for example, [36, Subsection 1.4.3] for an overview). A critical point of the Dirichlet energy, which corresponds to a standard harmonic map, is stable if the second variation is positive. Another variant of defining the stability of standard harmonic maps is to study the spectrum of the associated Jacobi operator. This operator is a linear second order elliptic operator and can be derived by linearizing the standard harmonic map equation.

In order to discuss the stability of harmonic maps with torsion we cannot consider the second variation of an energy functional. Nevertheless, we can calculate the linearization of the equation for harmonic maps with torsion and obtain a corresponding Jacobi operator.

Theorem 3.4. Let $\phi: M \rightarrow N$ be a smooth harmonic map with torsion. In terms of the Levi-Civita connection the corresponding Jacobi operator is given by

$$
J_{\phi}^{\mathrm{LC}}(\eta):=\Delta^{\mathrm{LC}} \eta+R^{N}(\eta, d \phi) d \phi+\left(\nabla_{\eta}^{\mathrm{LC}} A\right)(d \phi, d \phi)+A\left(\nabla^{\mathrm{LC}} \eta, d \phi\right)+A\left(d \phi, \nabla^{\mathrm{LC}} \eta\right) .
$$

Expressed via a metric connection with torsion the Jacobi-field equation acquires the form

$$
\begin{aligned}
J_{\phi}^{\mathrm{Tor}}(\eta):= & \Delta^{\mathrm{Tor}} \eta+R_{\text {Tor }}^{N}(\eta, d \phi) d \phi \\
& +\left(\nabla_{d \phi\left(e_{i}\right)}^{\mathrm{LC}} A\right)\left(\eta, d \phi\left(e_{i}\right)\right)+A\left(\nabla^{\mathrm{LC}} \eta, d \phi\right)+A(\eta, \tau(\phi))+A(d \phi, A(\eta, d \phi)) \\
& -\left(\nabla_{d \phi\left(e_{i}\right)}^{\mathrm{LC}} A\right)\left(d \phi\left(e_{i}\right), \eta\right)-A(\tau(\phi), \eta)-A\left(d \phi, \nabla^{\mathrm{LC}} \eta\right)-A(d \phi, A(d \phi, \eta)) .
\end{aligned}
$$

Proof. Suppose we have a smooth harmonic map with torsion, i.e., a solution of (1.3). To derive the associated Jacobi-field equation we consider a variation of the map $\phi$ defined by $\phi_{t}:(-\epsilon, \epsilon) \times M \rightarrow N$ satisfying $\left.d \phi_{t}\left(\partial_{t}\right)\right|_{t=0}=\eta$.

In order to obtain the Jacobi-field equation expressed via the Levi-Civita connection we calculate

$$
0=\left.\frac{\nabla}{\partial t}\left(\tau\left(\phi_{t}\right)+A\left(d \phi_{t}, d \phi_{t}\right)\right)\right|_{t=0} .
$$

It is well known that (see, for example, [36, Subsection 1.4.3])

$$
\left.\frac{\nabla}{\partial t} \tau\left(\phi_{t}\right)\right|_{t=0}=\Delta^{\mathrm{LC}} \eta+R^{N}(\eta, d \phi) d \phi
$$


Regarding the torsion endomorphism, using the Levi-Civita connection, we find

$$
\left.\frac{\nabla}{\partial t}\left(A\left(d \phi_{t}, d \phi_{t}\right)\right)\right|_{t=0}=\left(\nabla_{\eta}^{\mathrm{LC}} A\right)(d \phi, d \phi)+A\left(\nabla^{\mathrm{LC}} \eta, d \phi\right)+A\left(d \phi, \nabla^{\mathrm{LC}} \eta\right),
$$

which yields the first statement.

As a second step we derive the Jacobi-field equation making use of a metric connection with torsion. Thus, we again compute

$$
\begin{aligned}
0= & \left.\frac{\nabla}{\partial t} \tau^{\text {Tor }}(\phi)\right|_{t=0} \\
= & R_{\text {Tor }}^{N}(\eta, d \phi) d \phi+\left.\nabla_{e_{i}}^{\mathrm{Tor}} \nabla_{\partial_{t}}^{\text {Tor }} d \phi_{t}\left(e_{i}\right)\right|_{t=0} \\
= & R_{\text {Tor }}^{N}(\eta, d \phi) d \phi+\Delta^{\text {Tor }} \eta+\left.\nabla_{e_{i}}^{\text {Tor }}\left(T\left(d \phi_{t}\left(\partial_{t}\right), d \phi_{t}\left(e_{i}\right)\right)\right)\right|_{t=0} \\
= & R_{\text {Tor }}^{N}(\eta, d \phi) d \phi+\Delta^{\mathrm{Tor}} \eta+\left.\nabla_{e_{i}}^{\mathrm{LC}}\left(A\left(d \phi_{t}\left(\partial_{t}\right), d \phi_{t}\left(e_{i}\right)\right)\right)\right|_{t=0}+A(d \phi, A(\eta, d \phi)) \\
& -\left.\nabla_{e_{i}}^{\mathrm{LC}}\left(A\left(d \phi\left(e_{i}\right), d \phi_{t}\left(\partial_{t}\right)\right)\right)\right|_{t=0}-A(d \phi, A(d \phi, \eta))
\end{aligned}
$$

By a direct calculation we find

$$
\left.\nabla_{e_{i}}^{\mathrm{LC}}\left(A\left(d \phi_{t}\left(\partial_{t}\right), d \phi_{t}\left(e_{i}\right)\right)\right)\right|_{t=0}=\left(\nabla_{d \phi\left(e_{i}\right)}^{\mathrm{LC}} A\right)\left(\eta, d \phi\left(e_{i}\right)\right)+A\left(\nabla^{\mathrm{LC}} \eta, d \phi\right)+A(\eta, \tau(\phi))
$$

and a similar formula holds for the remaining term. The claim then follows from combining the different equations.

Remark 3.5. It is not hard to see that (3.9) and (3.10) can be easily transformed into each other. Using the formula for the connection Laplacians (3.4) we find

$$
\begin{aligned}
\Delta^{\mathrm{Tor}} \eta= & \Delta^{\mathrm{LC}} \eta+2 A\left(d \phi, \nabla^{\mathrm{LC}} \eta\right)+\left(\nabla_{d \phi\left(e_{i}\right)}^{\mathrm{LC}} A\right)\left(d \phi\left(e_{i}\right), \eta\right) \\
& +A(\tau(\phi), \eta)+A(d \phi, A(d \phi, \eta)) .
\end{aligned}
$$

Moreover, from (2.5) we obtain

$$
\begin{aligned}
R_{\mathrm{Tor}}^{N}(\eta, d \phi) d \phi= & R_{\mathrm{LC}}^{N}(\eta, d \phi) d \phi+\left(\nabla_{\eta}^{\mathrm{LC}} A\right)(d \phi, d \phi)-\left(\nabla_{d \phi\left(e_{i}\right)}^{\mathrm{LC}} A\right)\left(\eta, d \phi\left(e_{i}\right)\right) \\
& +A(\eta, A(d \phi, d \phi))-A(d \phi, A(\eta, d \phi)) .
\end{aligned}
$$

Inserting both identities into (3.10) then leads to (3.9).

As in the case of the standard Jacobi field equation both (3.9) and (3.10) constitute a linear elliptic differential operator of second order. As we assume the manifold $M$ to be closed we know that both

$J_{\phi}^{\mathrm{LC}}$ and $J_{\phi}^{\text {Tor }}$ have a discrete spectrum. For this reason we can in principle perform, as in the case of standard harmonic maps, a stability analysis of harmonic maps with torsion employing methods from spectral geometry. However, as we can define the Jacobi operator for either the Levi-Civita connection or for a connection with torsion, there does not seem to be a unique way of defining the stability of a harmonic map with torsion.

It is well known that standard harmonic maps from closed domains to target spaces with negative curvature are stable. From a spectral point of view this is reflected in the fact that the corresponding Jacobi operator does not have any negative eigenvalues. If we carry out the same analysis for harmonic maps with torsion then it is hard to make a general statement on the spectrum of the Jacobi operator. Consequently, in general, we cannot conclude that harmonic maps with torsion to target spaces with negative curvature (both defined for the Levi-Civita connection or for a connection with torsion) are stable.

In summary, we can say that it is very difficult to understand the stability of harmonic maps with torsion such that this topic deserves a thorough future investigation.

\section{Analytic aspects of harmonic maps with torsion}

This section is devoted to various analytic aspects of a given harmonic map with torsion. 


\subsection{The unique continuation property for harmonic maps with torsion}

It was proved by Sampson [31, Theorem 2] that standard harmonic maps satisfy the unique continuation property and we now extend this result to harmonic maps with torsion. To this end we recall the following theorem (see [5, p. 248]).

Theorem 4.1 (See [5]). Let $A$ be a linear elliptic second-order differential operator defined on a domain $D$ of $\mathbb{R}^{m}$. Let $u=\left(u^{1}, \ldots, u^{m}\right)$ be functions in $D$ satisfying the inequality

$$
\left|A u^{\alpha}\right| \leqslant C\left(\sum_{i, \alpha}\left|\frac{\partial u^{\alpha}}{\partial x^{i}}\right|+\sum_{\alpha}\left|u^{\alpha}\right|\right) .
$$

If $u=0$ in an open set, then $u=0$ throughout $D$.

Using this result we can prove the following theorem.

Theorem 4.2. Let $\phi, \phi^{\prime} \in C^{2}(M, N)$ be two harmonic maps with torsion. If $\phi$ and $\phi^{\prime}$ are equal on a connected open set $W$ of $M$ then they coincide on the whole connected component of $M$ which contains $W$.

Proof. Let $\left(U, x^{i}\right)$ be a coordinate ball on $M$. By shrinking $U$ if necessary we can assume that both $\phi$ and $\phi^{\prime}$ map $U$ into a single coordinate chart $\left(V, y^{\alpha}\right)$ of $N$. We set $u^{\alpha}:=\phi^{\alpha}-\phi^{\alpha}$ and using the local form of the Euler-Lagrange equation (2.7) we find

$$
\begin{aligned}
\Delta u^{\alpha}= & -\Gamma_{\beta \gamma}^{\alpha}(\phi) \frac{\partial \phi^{\beta}}{\partial x^{i}} \frac{\partial \phi^{\gamma}}{\partial x^{j}} g^{i j}+\Gamma_{\beta \gamma}^{\alpha}\left(\phi^{\prime}\right) \frac{\partial \phi^{\prime \beta}}{\partial x^{i}} \frac{\partial \phi^{\prime \gamma}}{\partial x^{j}} g^{i j} \\
& -A_{\beta \gamma}^{\alpha}(\phi) \frac{\partial \phi^{\beta}}{\partial x^{i}} \frac{\partial \phi^{\gamma}}{\partial x^{j}} g^{i j}+A_{\beta \gamma}^{\alpha}\left(\phi^{\prime}\right) \frac{\partial \phi^{\prime \beta}}{\partial x^{i}} \frac{\partial \phi^{\prime \gamma}}{\partial x^{j}} g^{i j} \\
= & -\left(\Gamma_{\beta \gamma}^{\alpha}(\phi)-\Gamma_{\beta \gamma}^{\alpha}\left(\phi^{\prime}\right)\right) \frac{\partial \phi^{\beta}}{\partial x^{i}} \frac{\partial \phi^{\gamma}}{\partial x^{j}} g^{i j}-\Gamma_{\beta \gamma}^{\alpha}\left(\phi^{\prime}\right) \frac{\partial u^{\beta}}{\partial x^{i}} \frac{\partial \phi^{\gamma}}{\partial x^{j}} g^{i j}-\Gamma_{\beta \gamma}^{\alpha}\left(\phi^{\prime}\right) \frac{\partial \phi^{\beta}}{\partial x^{i}} \frac{\partial u^{\gamma}}{\partial x^{j}} g^{i j} \\
& -\left(A_{\beta \gamma}^{\alpha}(\phi)-A_{\beta \gamma}^{\alpha}\left(\phi^{\prime}\right)\right) \frac{\partial \phi^{\beta}}{\partial x^{i}} \frac{\partial \phi^{\gamma}}{\partial x^{j}} g^{i j}-A_{\beta \gamma}^{\alpha}\left(\phi^{\prime}\right) \frac{\partial u^{\beta}}{\partial x^{i}} \frac{\partial \phi^{\gamma}}{\partial x^{j}} g^{i j}-A_{\beta \gamma}^{\alpha}\left(\phi^{\prime}\right) \frac{\partial \phi^{\beta}}{\partial x^{i}} \frac{\partial u^{\gamma}}{\partial x^{j}} g^{i j} \\
\leqslant & C\left(\sum_{i, \alpha}\left|\frac{\partial u^{\alpha}}{\partial x^{i}}\right|+\sum_{\alpha}\left|u^{\alpha}\right|\right),
\end{aligned}
$$

where we used the mean-value inequality to estimate the terms involving the Christoffel symbols and the torsion endomorphisms. Moreover, we utilized that $\phi$ and its derivatives are bounded as we are considering maps between compact sets. The result now follows from application of Theorem 4.1.

Remark 4.3. Note that the same proof from above would also work for the VT-harmonic maps (1.4) which are a variant of harmonic maps with torsion that also contain a term that is linear in $d \phi$ on the right-hand side.

\subsection{Regularity of weak solutions}

In this subsection we establish the regularity of weak harmonic maps with torsion. It turns out that the additional nonlinearity arising due to the non-vanishing torsion has the right antisymmetric structure such that the known regularity theory for standard harmonic maps is still applicable.

First, we define a weak solution of (1.3).

Definition 4.4. We call a map $\phi \in W^{1,2}(M, N)$ a weak harmonic map with torsion if it solves (1.3) in the sense of distributions.

We will prove the following regularity result for weak harmonic maps with torsion.

Theorem 4.5. Let $(M, g)$ be a closed Riemannian manifold with $\operatorname{dim} M=m$ and $(N, h)$ be a compact Riemannian manifold. Suppose that $\phi: M \rightarrow N$ is a weak solution of (1.3) that satisfies the Morrey growth condition

$$
\sup _{x \in U}\left(\frac{1}{r^{m-2}} \int_{B_{r}(x) \cap U}|d \phi|^{2} d \mu\right)^{\frac{1}{2}}<\epsilon
$$


for some small $\epsilon>0$.

Then a weak solution $\phi \in W^{1,2}(M, N)$ is smooth in $U$, where $U$ is an open subset of $M$.

Remark 4.6. (1) In the case where $M$ is a closed surface it is enough to demand that $\phi \in W^{1,2}(M, N)$ as we can always find a small disc on which (4.2) holds.

(2) In the regularity analysis of standard harmonic maps one usually demands that the harmonic map is stationary which is a weaker condition than (4.2). A harmonic map is stationary if it is a critical point of the energy $E(\phi)$ both with respect to variations of the map and with respect to variations of the metric on the domain. The condition of being stationary can be interpreted as a weak formulation of the invariance of the energy under diffeomorphisms on the domain [11, Subsection 2.3]. As harmonic maps with torsion do not allow for a variational formulation we cannot come up with a generalization of the notion of being stationary but have to demand the smallness condition (4.2).

In order to prove Theorem 4.5 it is natural to apply the embedding theorem of Nash and to isometrically embed the target manifold $N$, where $n:=\operatorname{dim} N$, into some $\mathbb{R}^{q}$ of sufficiently large dimension $q$. We denote this isometric embedding by $\iota: N \rightarrow \mathbb{R}^{q}$ which we assume to be smooth. Now, we consider the composite map $\phi^{\prime}:=\iota \circ \phi: M \rightarrow \mathbb{R}^{q}$ and let $u^{\alpha}, 1 \leqslant \alpha \leqslant q$ be global coordinates on the ambient space $\mathbb{R}^{q}$. Moreover, let $\nu_{\theta}, \theta=n+1, \ldots, q$ be an orthonormal frame of the submanifold $\iota(\phi)$. In the following we will still write $\phi$ instead of $\phi^{\prime}$ in order to shorten the notation.

Lemma 4.7. The extrinsic version of the equation for harmonic maps with torsion (1.3) for $\phi: M \rightarrow$ $\mathbb{R}^{q}$ is given by

$$
-\Delta \phi^{\alpha}=\left(\omega_{i}^{\alpha \beta}+B_{i}^{\alpha \beta}\right) \frac{\partial \phi^{\beta}}{\partial x^{i}},
$$

where $1 \leqslant \alpha \leqslant q$ and

$$
\begin{aligned}
\omega_{i}^{\alpha \beta} & :=\frac{\partial \phi^{\gamma}}{\partial x^{i}} \frac{\partial \nu_{l}^{\beta}}{\partial u^{\gamma}} \nu_{l}^{\alpha}-\frac{\partial \phi^{\gamma}}{\partial x^{i}} \frac{\partial \nu_{l}^{\alpha}}{\partial u^{\gamma}} \nu_{l}^{\beta}=-\omega_{i}^{\alpha \beta}, \\
B_{i}^{\alpha \beta} & :=A_{\gamma \beta}^{\alpha} \frac{\partial \phi^{\gamma}}{\partial x^{i}}=-B_{i}^{\beta \alpha} .
\end{aligned}
$$

Proof. It is well known by now that the standard harmonic equation can be written in the form (4.3) (see, for example, the discussion in the introduction of [28]). We can extend the torsion endomorphism $A$ to the ambient space by parallel transport. Moreover, we set

$$
A_{\gamma \beta}^{\alpha}:=h^{\alpha \delta} A_{\gamma \beta \delta}=h^{\alpha \delta}\left\langle A\left(\partial_{y^{\gamma}}, \partial_{y^{\beta}}\right), \partial_{y^{\delta}}\right\rangle,
$$

where $\partial_{y^{\alpha}}$ is a local basis of $T N$. The antisymmetry follows from (2.3).

Now, we recall the following regularity result. Suppose that we have a distributional solution to

$$
-\Delta u=\Omega \cdot \nabla u,
$$

where $u: B^{m} \rightarrow \mathbb{R}^{q}$ and $\Omega$ is antisymmetric. Here, $B^{m}$ denotes the $m$-dimensional unit disc.

For such kind of systems the following regularity result holds [29, Theorem 1.1].

Theorem 4.8. For every $m \in \mathbb{N}$ there exists an $\epsilon(m)>0$ such that for every $\Omega \in L^{2}\left(B^{m}, \mathfrak{s o}(q) \otimes \mathbb{R}^{m}\right)$ and for every weak solution $u \in W^{1,2}\left(B^{m}, \mathbb{R}^{q}\right)$ of (4.4) that satisfies the Morrey growth condition

$$
\sup _{x \in B}\left(\frac{1}{r^{m-2}} \int_{B_{r}(x) \cap B}\left(|\nabla u|^{2}+|\Omega|^{2}\right) d \mu\right)^{\frac{1}{2}}<\epsilon(m),
$$

we have that $u$ is locally Hölder-continuous in $B^{m}$ with the exponent $0<\alpha=\alpha(m)<1$.

We are now able to give the proof of Theorem 4.5.

Proof of Theorem 4.5. Thanks to (4.3) the equation for harmonic maps with torsion can be written in the form (4.4). In addition, as $N$ is compact by assumption and since $\phi$ is a weak solution of (1.3) we 
have $\Omega \in L^{2}\left(B^{m}, \mathfrak{s o}(q) \otimes \mathbb{R}^{m}\right)$. As also the smallness condition (4.2) holds by assumption we can apply Theorem 4.8 to the extrinsic version of the equation for harmonic maps with torsion (4.3) yielding the local Hölder continuity of $\phi$. Now, the same bootstrap argument as for standard harmonic maps shows that $\phi$ must be smooth (see, for example, [22, Theorem 7.4.1]).

\subsection{Local energy estimates and a removable singularity theorem}

A classic result of Sacks and Uhlenbeck [30] shows that standard harmonic maps of finite Dirichlet energy from a two-dimensional domain cannot have isolated point singularities or phrased differently isolated point singularities can always be removed. One should expect that such a result also holds for harmonic maps with torsion as their nonlinear structure on the right-hand side is the same as in the case of standard harmonic maps.

However, it turns out that the method of proof applied by Sacks and Uhlenbeck does not directly carry over to harmonic maps with torsion. At the heart of their proof is a Pohozaev identity which is derived from the stress-energy tensor. As harmonic maps with torsion do not arise from a variational principle it is not possible to extend the methods used in the analysis of standard harmonic maps and we have to make use of a different method of proof here.

In the following it will again turn out to be useful to apply the following extrinsic version of the equation for harmonic maps with torsion

$$
-\Delta \phi=\mathbb{I}(d \phi, d \phi)+A(d \phi, d \phi) .
$$

Here, $\phi: M \rightarrow \mathbb{R}^{q}$ is a vector-valued map and II denotes the second fundamental form of the embedding into the ambient space $\mathbb{R}^{q}$. To obtain this extrinsic version of the equation for harmonic maps with torsion one again applies the embedding theorem of Nash (see the discussion in front of Lemma 4.7 and the introduction of [28] for more details).

Note that harmonic maps with torsion have the same analytic structure as standard harmonic maps in the sense that they satisfy

$$
|\Delta \phi| \leqslant C|d \phi|^{2}
$$

for a positive constant $C>0$.

This suggests to define the following local energy.

Definition 4.9. The local energy of a map $\phi: M \rightarrow N$ is defined as follows:

$$
E(\phi, U)=\int_{U}|d \phi|^{2} d \mu
$$

where $U \subset M$.

We obtain the following result.

Theorem 4.10 (Removable singularity theorem). Let $(M, g)$ be a closed Riemannian manifold of dimension $\operatorname{dim} M=2$ and $(N, h)$ be a compact Riemannian manifold. Let $\phi$ be a harmonic map with torsion which is smooth on $U \backslash\{p\}$ for some $p \in U \subset M$. If $\phi$ has finite energy $E(\phi, U)$, then $\phi$ extends to a smooth solution on $U$.

In order to prove Theorem 4.10 we set $D^{\prime}=D \backslash\{0\}$, where $D \subset M$ denotes the unit disc in two dimensions, and make use of the following lemma.

Lemma 4.11. Let $(M, g)$ be a closed Riemannian manifold of dimension $\operatorname{dim} M=2$ and suppose $\phi \in W^{1,2}\left(D^{\prime}, \mathbb{R}^{q}\right)$ satisfies

$$
\int_{D^{\prime}}\langle\nabla \phi, \nabla \eta\rangle d \mu=\int_{D^{\prime}} f(x, \phi, \nabla \phi) \eta d \mu
$$

for all $\eta \in W_{0}^{1,2} \cap L^{\infty}\left(D^{\prime}, \mathbb{R}^{q}\right)$, where $f$ satisfies the growth assumption

$$
|f(x, \phi, p)| \leqslant a+b|p|^{2}
$$


with constants $a, b>0$ for all $(x, \phi, p) \in D^{\prime} \times \mathbb{R}^{q} \times \mathbb{R}^{2 q}$. Then we also have

$$
\int_{D}\langle\nabla \phi, \nabla \eta\rangle d \mu=\int_{D} f(x, \phi, \nabla \phi) \eta d \mu .
$$

Proof. For a proof, see [21, Lemma A.2, p. 225].

We can now give the proof of Theorem 4.10 .

Proof of Theorem 4.10. First of all, we note that (4.8) is the weak version of (4.6). Hence, due to the assumption $E(\phi, U)<\infty$ we can apply Lemma 4.11. Thus, we know that every weak harmonic map with torsion defined on a disc with the origin removed can be extended to a weak harmonic map with torsion on the whole unit disc. However, the assumptions of Theorem 4.10 allow us to also apply the regularity result Theorem 4.5 such that all the weak solutions considered here are actually smooth.

Besides the removable singularity theorem we can also give the following local energy estimates.

Theorem 4.12 ( $\epsilon$-regularity theorem). Assume that $\phi$ is a smooth harmonic map with torsion with small energy

$$
E(\phi, D)<\epsilon
$$

Then the following estimate holds:

$$
\|d \phi\|_{W^{1, p}(\tilde{D})} \leqslant C(\tilde{D}, p)\|d \phi\|_{L^{2}(D)}
$$

for all $\tilde{D} \subset D, p>1$, where $C(\tilde{D}, p)$ is a positive constant depending only on $\tilde{D}$ and $p$.

Proof. This can be proved by the same method as for standard harmonic maps (see, for example, [21, Lemma 2.4.1] or [7, Theorem 3.3]).

Exploiting the scaling invariance of (4.6) we also obtain the following corollary.

Corollary 4.13. There is an $\epsilon>0$ small enough such that if $\phi$ is a smooth harmonic map with torsion with small energy $E(\phi, D)<\epsilon$ then for any $x \in D_{\frac{1}{2}}$ we have

$$
|d \phi(x)||x| \leqslant C\|d \phi\|_{L^{2}\left(D_{2|x|}\right)}
$$

where $C$ is a positive constant.

Proof. This follows from a scaling argument. Fix any $x_{0} \in D \backslash\{0\}$ and define $\tilde{\phi}$ as

$$
\tilde{\phi}(x):=\phi\left(x_{0}+\left|x_{0}\right| x\right) .
$$

It is easy to see that $\tilde{\phi}$ is a smooth solution of (4.6) on $D$ with $E(\tilde{\phi}, D)<\epsilon$. By application of Theorem 4.12, we have

$$
|d \tilde{\phi}|_{L^{\infty}\left(D_{\frac{1}{2}}\right)} \leqslant C\|d \tilde{\phi}\|_{L^{2}(D)}
$$

and scaling back yields the assertion.

\subsection{A Liouville theorem on complete non-compact manifolds}

In this subsection, we establish a vanishing result for solutions of (1.3) on a large class of complete noncompact domain manifolds. For standard harmonic maps a corresponding result was obtained in [25] (see also [10] for further generalizations).

At the heart of the proof is a Euclidean type Sobolev inequality, i.e.,

$$
\left(\int_{M}|u|^{2 m /(m-2)} \operatorname{dvol}_{g}\right)^{\frac{m-2}{m}} \leqslant C_{2} \int_{M}|\nabla u|^{2} \operatorname{dvol}_{g}
$$


for all $u \in W^{1,2}(M)$ with compact support, where $C_{2}$ is a positive constant that depends on the geometry of $M$. Such an inequality holds in $\mathbb{R}^{m}$ and is well known as the Gagliardo-Nirenberg inequality. However, if one considers a non-compact complete Riemannian manifold of infinite volume one has to make additional assumptions to have an equality of the form (4.11) at hand (see the introduction of [10] for more details).

We will make use of a cutoff function $0 \leqslant \eta \leqslant 1$ on $M$ that satisfies

$$
\eta(x)=1 \quad \text { for } x \in B_{R}\left(x_{0}\right), \quad \eta(x)=0 \quad \text { for } x \in B_{2 R}\left(x_{0}\right), \quad|\nabla \eta| \leqslant \frac{C}{R} \quad \text { for } x \in M,
$$

where $B_{R}\left(x_{0}\right)$ denotes the geodesic ball around $x_{0}$ with the radius $R$.

Finally, we obtain the following Liouville theorem.

Theorem 4.14. Let $(M, g)$ be a complete and non-compact Riemannian manifold of dimension $\operatorname{dim} M$ $=m>2$ with positive Ricci curvature that admits a Euclidean type Sobolev inequality of the form (4.11). Moreover, let $N$ be a Riemannian manifold of bounded geometry, i.e.,

$$
\left|R^{N}\right|_{L^{\infty}}+|A|_{L^{\infty}}<\infty .
$$

Here, $A$ denotes the torsion endomorphism defined in (2.4).

Assume that $\phi$ is a smooth solution of (1.3). If

$$
\int_{M}|d \phi|^{m} \operatorname{dvol}_{g}<\epsilon
$$

where $\epsilon>0$ satisfies

$$
\epsilon \leqslant\left[\frac{2 m-4}{m^{2} C_{s}}\left(\left|R^{N}\right|_{L^{\infty}}+|A|_{L^{\infty}} \frac{1+m}{2}\right)^{-1}\right]^{\frac{m}{2}}
$$

then $\phi$ must be constant.

First, we will derive the following inequality similar to [10, Lemma 2.2].

Lemma 4.15. Suppose that $\phi: M \rightarrow N$ is a smooth solution of (1.3) and $\operatorname{dim} M>2$. Then the following inequality holds:

$$
\begin{aligned}
\frac{C}{R^{2}} \int_{M}|d \phi|^{m} \operatorname{dvol}_{g} \geqslant & \int_{M} \eta^{2}\left\langle d \phi\left(\operatorname{Ric}^{M}\right), d \phi\right\rangle|d \phi|^{m-2} \operatorname{dvol}_{g}+\frac{1}{2} \int_{M} \eta^{2}|\nabla d \phi|^{2}|d \phi|^{m-2} \operatorname{dvol}_{g} \\
& +\left.\left.\frac{2 m-4}{m^{2}} \int_{M} \eta^{2}|d| d \phi\right|^{\frac{m}{2}}\right|^{2} \operatorname{dvol}_{g} \\
& -\left(\left|R^{N}\right|_{L^{\infty}}+\frac{1+m}{2}|A|_{L^{\infty}}\right) \int_{M} \eta^{2}|d \phi|^{m+2} \operatorname{dvol}_{g}
\end{aligned}
$$

Proof. Testing the Bochner formula for the Levi-Civita connection (3.1) with $\eta^{2}|d \phi|^{m-2} d \phi\left(e_{i}\right)$ and integrating over $M$ we find

$$
\begin{aligned}
\int_{M} \eta^{2}\langle\Delta d \phi, d \phi\rangle|d \phi|^{m-2} \operatorname{dvol}_{g}= & \int_{M} \eta^{2}\left\langle d \phi\left(\operatorname{Ric}^{M}\left(e_{i}\right)\right), d \phi\left(e_{i}\right)\right\rangle|d \phi|^{m-2} \operatorname{dvol}_{g} \\
& +\int_{M} \eta^{2}\left\langle R^{N}\left(d \phi\left(e_{i}\right), d \phi\left(e_{j}\right)\right) d \phi\left(e_{i}\right), d \phi\left(e_{j}\right)\right\rangle|d \phi|^{m-2} \operatorname{dvol}_{g} \\
& +\int_{M} \eta^{2}\langle\nabla \tau(\phi), d \phi\rangle|d \phi|^{m-2} \operatorname{dvol}_{g} .
\end{aligned}
$$

Using integration by parts we may rewrite

$$
\begin{aligned}
\int_{M} \eta^{2}\langle\nabla \tau(\phi), d \phi\rangle|d \phi|^{m-2} \operatorname{dvol}_{g}= & -2 \int_{M} \eta \nabla \eta\langle\tau(\phi), d \phi\rangle|d \phi|^{m-2} \operatorname{dvol}_{g} \\
& -\int_{M} \eta^{2}|\tau(\phi)|^{2}|d \phi|^{m-2} \operatorname{dvol}_{g}
\end{aligned}
$$




$$
-(m-2) \int_{M} \eta^{2}\langle\tau(\phi), d \phi\rangle|d \phi|^{m-4}\langle d \phi, \nabla d \phi\rangle \operatorname{dvol}_{g}
$$

and also

$$
\begin{aligned}
\int_{M} \eta^{2}\langle\Delta d \phi, d \phi\rangle|d \phi|^{m-2} \operatorname{dvol}_{g}= & -2 \int_{M} \eta \nabla \eta\langle\nabla d \phi, d \phi\rangle|d \phi|^{m-2} \mathrm{dvol}_{g}-\int_{M} \eta^{2}|\nabla d \phi|^{2}|d \phi|^{m-2} \mathrm{dvol}_{g} \\
& -(m-2) \int_{M} \eta^{2}|\langle\nabla d \phi, d \phi\rangle|^{2}|d \phi|^{m-4} \operatorname{dvol}_{g} .
\end{aligned}
$$

This allows us to deduce the following inequality:

$$
\begin{aligned}
& \int_{M} \eta^{2}\left\langle d \phi\left(\operatorname{Ric}^{M}\left(e_{i}\right)\right), d \phi\left(e_{i}\right)\right\rangle|d \phi|^{m-2} \operatorname{dvol}_{g}+\int_{M} \eta^{2}|\nabla d \phi|^{2}|d \phi|^{m-2} \mathrm{dvol}_{g} \\
&+(m-2) \int_{M} \eta^{2}|\langle\nabla d \phi, d \phi\rangle|^{2}|d \phi|^{m-4} \operatorname{dvol}_{g} \\
& \leqslant \frac{C}{R^{2}} \int_{M}|d \phi|^{m} \operatorname{dvol}_{g}+\frac{1}{2} \int_{M} \eta^{2}|\nabla d \phi|^{2}|d \phi|^{m-2} \operatorname{dvol}_{g}+\frac{1+m}{2} \int_{M} \eta^{2}|\tau(\phi)|^{2}|d \phi|^{m-2} \operatorname{dvol}_{g} \\
& \quad+\left.\left|R^{N} L_{L^{\infty}} \int_{M} \eta^{2}\right| d \phi\right|^{m+2} \operatorname{dvol}_{g}+\frac{m-2}{2} \int_{M} \eta^{2}|\langle\nabla d \phi, d \phi\rangle|^{2}|d \phi|^{m-4} \operatorname{dvol}_{g} .
\end{aligned}
$$

The preceding inequality yields

$$
\begin{aligned}
& \int_{M} \eta^{2}\left\langle d \phi\left(\operatorname{Ric}^{M}\left(e_{i}\right)\right), d \phi\left(e_{i}\right)\right\rangle|d \phi|^{m-2} \operatorname{dvol}_{g}+\frac{1}{2} \int_{M} \eta^{2}|\nabla d \phi|^{2}|d \phi|^{m-2} \mathrm{dvol}_{g} \\
& +\frac{m-2}{2} \int_{M} \eta^{2}|\langle\nabla d \phi, d \phi\rangle|^{2}|d \phi|^{m-4} \operatorname{dvol}_{g} \\
& \quad \leqslant \frac{C}{R^{2}} \int_{M}|d \phi|^{m} \operatorname{dvol}_{g}+\frac{1+m}{2}+\left(\left|R^{N}\right|_{L^{\infty}}+\frac{1+m}{2}|A|_{L^{\infty}}\right) \int_{M} \eta^{2}|d \phi|^{m+2} \mathrm{dvol}_{g},
\end{aligned}
$$

where we have used that $\phi$ is a solution of (1.3) in the last step. The claim follows from using

$$
|\langle\nabla d \phi, d \phi\rangle|^{2}|d \phi|^{m-4}=\left.\left.\frac{1}{4}|d| d \phi\right|^{2}\right|^{2}|d \phi|^{m-4}=\left.\left.\frac{4}{m^{2}}|d| d \phi\right|^{\frac{m}{2}}\right|^{2} .
$$

This completes the proof.

To complete the proof we also need the following lemma.

Lemma 4.16. Let $(M, g)$ be a complete non-compact Riemannian manifold of infinite volume that admits a Euclidean type Sobolev inequality. Assume that $f$ is a positive function on $M$. For $\operatorname{dim} M=$ $m>2$ the following inequality holds:

$$
\int_{M} \eta^{2} f^{m+2} \operatorname{dvol}_{g} \leqslant C_{s}\left(\int_{M} f^{m} \operatorname{dvol}_{g}\right)^{\frac{2}{m}}\left(\frac{1}{R^{2}} \int_{M} f^{m} \operatorname{dvol}_{g}+\int_{M} \eta^{2}\left|d f^{\frac{m}{2}}\right|^{2} \mathrm{dvol}_{g}\right),
$$

where the positive constant $C_{s}$ depends on $m$ and the geometry of $M$.

Proof. For a derivation see the proof of [10, Lemma 2.4].

At this point we are ready to complete the proof of Theorem 4.14.

Proof of Theorem 4.14. We apply (4.14) to the last term on the right-hand side of (4.13) with $f=|d \phi|$ and by choosing $\epsilon$ small enough and taking the limit $R \rightarrow \infty$ we obtain from (4.13) that

$$
0 \geqslant \int_{M}\left\langle d \phi\left(\operatorname{Ric}^{M}\right), d \phi\right\rangle|d \phi|^{m-2} \operatorname{dvol}_{g} .
$$

By assumption $M$ has positive Ricci curvature and thus $\operatorname{Ric}^{M}$ is a positive definite non-degenerate bilinear form on $T M$. As $m>2$ this completes the proof. 
Acknowledgements The author gratefully acknowledges the support of the Austrian Science Fund (FWF) through the project P30749-N35 "Geometric Variational Problems from String Theory".

Open access funding provided by Austrian Science Fund (FWF).

Open Access This article is licensed under a Creative Commons Attribution 4.0 International License, which permits use, sharing, adaptation, distribution and reproduction in any medium or format, as long as you give appropriate credit to the original author(s) and the source, provide a link to the Creative Commons licence, and indicate if changes were made.

The images or other third party material in this article are included in the article's Creative Commons licence, unless indicated otherwise in a credit line to the material. If material is not included in the article's Creative Commons licence and your intended use is not permitted by statutory regulation or exceeds the permitted use, you will need to obtain permission directly from the copyright holder.

To view a copy of this licence, visit http://creativecommons.org/licenses/by/4.0/

\section{References}

1 Agricola I. Connections on naturally reductive spaces, their Dirac operator and homogeneous models in string theory. Comm Math Phys, 2003, 232: 535-563

2 Agricola I. The Srní lectures on non-integrable geometries with torsion. Arch Math Brno, 2006, 42: 5-84

3 Agricola I, Kraus M. Manifolds with vectorial torsion. Differential Geom Appl, 2016, 45: 130-147

4 Agricola I, Thier C. The geodesics of metric connections with vectorial torsion. Ann Global Anal Geom, 2004, 26: $321-332$

5 Aronszajn N. A unique continuation theorem for solutions of elliptic partial differential equations or inequalities of second order. J Math Pures Appl (9), 1957, 36: 235-249

6 Branding V. Dirac-harmonic maps with torsion. Commun Contemp Math, 2016, 18: 1550064

7 Branding V. Energy estimates for the supersymmetric nonlinear sigma model and applications. Potential Anal, 2016, 45: $737-754$

8 Branding V. The heat flow for the full bosonic string. Ann Global Anal Geom, 2016, 50: 347-365

9 Branding V. A global weak solution to the full bosonic string heat flow. J Evol Equ, 2018, 18: 1819-1841

10 Branding V. A vanishing result for the supersymmetric nonlinear sigma model in higher dimensions. J Geom Phys, 2018, 134: 1-10

11 Branding V. The stress-energy tensor for polyharmonic maps. Nonlinear Anal, 2020, 190: 111616

12 Branding V, Hanisch F. Magnetic geodesics via the heat flow. Asian J Math, 2017, 21: 995-1014

13 Branding V, Kröncke K. The Ricci flow with metric torsion on closed surfaces. J Geom Anal, 2017, 27: 2098-2117

14 Cartan E. Sur les variétés à connexion affine, et la théorie de la relativité généralisée (première partie) (Suite). Ann Sci École Norm Sup (3), 1924, 41: 1-25

15 Chen Q, Jost J, Qiu H B. On VT-harmonic maps. Ann Global Anal Geom, 2020, 57: 71-94

16 Chong T, Dong Y X, Ren Y B, et al. On harmonic and pseudoharmonic maps from pseudo-Hermitian manifolds. Nagoya Math J, 2019, 234: 170-210

17 Dragomir S, Tomassini G. Differential Geometry and Analysis on CR Manifolds. Progress in Mathematics, vol. 246. Boston: Birkhäuser, 2006

18 Gauduchon P. La 1-forme de torsion d'une variété hermitienne compacte. Math Ann, 1984, 267: 495-518

19 Gauduchon P. Hermitian connections and Dirac operators. Boll Unione Mat Ital (9), 1997, 11: 257-288

20 Hélein F, Wood J C. Harmonic maps. In: Handbook of Global Analysis, vol. 1213. Amsterdam: Elsevier, 2008, $417-491$

21 Jost J. Two-Dimensional Geometric Variational Problems. Chichester: Wiley-Interscience, 1991

22 Jost J. Riemannian Geometry and Geometric Analysis, 6th ed. Heidelberg: Springer, 2011

23 Lin F H, Wang C Y. The Analysis of Harmonic Maps and Their Heat Flows. Hackensack: World Scientific, 2008

24 Morrow J, Kodaira K. Complex Manifolds. Providence: Amer Math Soc, 2006

25 Nakauchi N, Takakuwa S. A remark on p-harmonic maps. Nonlinear Anal, 1995, 25: 169-185

26 Pfäffle F, Stephan C A. On gravity, torsion and the spectral action principle. J Funct Anal, 2012, 262: 1529-1565

27 Ren Y B, Yang G L. Pseudo-harmonic maps from closed pseudo-Hermitian manifolds to Riemannian manifolds with nonpositive sectional curvature. Calc Var Partial Differential Equations, 2018, 57: 128

28 Rivière T. Conservation laws for conformally invariant variational problems. Invent Math, 2007, 168: 1-22

29 Rivière T, Struwe M. Partial regularity for harmonic maps and related problems. Comm Pure Appl Math, 2008, 61: 451-463

30 Sacks J, Uhlenbeck K. The existence of minimal immersions of 2-spheres. Ann of Math (2), 1981, 113: 1-24 
31 Sampson J H. Some properties and applications of harmonic mappings. Ann Sci École Norm Sup (4), 1978, 11: 211-228

32 Shapiro I L. Physical aspects of the space-time torsion. Phys Rep, 2002, 357: 113-213

33 Tanaka N. A Differential Geometric Study on Strongly Pseudo-Convex Manifolds. Lectures in Mathematics, No. 9. Tokyo: Kinokuniya Book-Store, 1975

34 Tricerri F, Vanhecke L. Homogeneous Structures on Riemannian Manifolds. London Mathematical Society Lecture Note Series, vol. 83. Cambridge: Cambridge University Press, 1983

35 Webster S M. Pseudo-Hermitian structures on a real hypersurface. J Differential Geom, 1978, 13: 25-41

36 Xin Y L. Geometry of Harmonic Maps. Progress in Nonlinear Differential Equations and Their Applications, vol. 23. Boston: Birkhäuser, 1996 